\title{
Retrospective Review of the Efficacy and Safety of Repeated Pulsed and Continuous Radiofrequency Lesioning of the Dorsal Root Ganglion/Segmental Nerve for Lumbar Radicular Pain
}

\author{
Jyotsna V. Nagda, MD, Craig W. Davis, MD, Zahid H. Bajwa, MD, and
}

Thomas T. Simopoulos, MD

From: Beth Israel Deaconess Medical Center, Harvard Medical School, Boston, MA

Dr. Nagda is an Instructor, Department of Anesthesia, Critical

Care, and Pain Medicine, Beth Israel Deaconess Medical Center, Harvard Medical School, Boston,

Dr. Davis is is a Fellow at the Beth Israel Deaconess Medical Center, Harvard Medical School, Boston,

MA.

Dr. Bajwa is an Associate Professor and Staff Physician, Department of Anesthesiology and Critical Care,

Beth Israel Deaconess Medical

Center, Harvard Medical School, Boston, MA.

Dr. Simopoulos is Assistant Professor, Department of Anesthesia, Critical Care, and Pain Medicine, Beth Israel Deaconess Medical Center, Harvard Medical School, Boston, MA.

Address correspondence: Jyotsna V. Nagda, MD

Beth Israel Deaconess Medical Center

330 Brookline Ave. Boston, MA 02215 E-mail: jnagda@bidmc.harvard. edu

Disclaimer: There was no external funding in the preparation of this manuscript. Conflict of interest: None.

Manuscript received: 02/14/2011 Revised manuscript received: 05/02/2011

Accepted for publication: 05/25/2011

Free full manuscript: www.painphysicianjournal.com
Background: Chronic lumbosacral radicular pain is a common source of radiating leg pain seen in pain management patients. These patients are frequently managed conservatively with multiple modalities including medications, physical therapy, and epidural steroid injections. Radiofrequency has been used to treat chronic radicular pain for over 30 years; however, there is a paucity of literature about the safety and efficacy of repeat radiofrequency lesioning.

Objectives: To determine the safety, success rate, and duration of pain relief of repeat pulsed radiofrequency (PRF) and continuous radiofrequency (CRF) lesioning of the dorsal root ganglion (DRG)/ sacral segmental nerves (SN) in patients with chronic lumbosacral radicular pain.

Study Design: Retrospective chart review

Setting: Outpatient multidisciplinary pain center

Methods: Medical record review of patients who were treated with pulsed and continuous radiofrequency lesioning of the lumbar dorsal root ganglia and segmental nerves and who reported initial success were evaluated for recurrence of pain and repeat radiofrequency treatment. Responses to subsequent treatments were compared to initial treatments for success rates, average duration of relief, and adverse neurologic side-effects.

Limitations: Retrospective chart review without a control group.

Results: Twenty-six women and 24 men were identified who received $50 \%$ pain relief or better after PRF and CRF of the lumbar DRG/ sacral SN for lumbosacral radicular pain. The mean age was 62 years (range, 25-86). The mean duration of relief for the 40 patients who had 2 treatments was 4.7 months (range 0-24; Se [standard error] 0.74). Twenty-eight patients had 3 treatments with an average duration of relief of 4.5 months (range 0-19 months; Se 0.74). Twenty patients had 4 treatments with a mean duration of relief of 4.4 months (range $0.5-18$; Se 0.95 ) and 18 patients who had 5 or more treatments received an average duration of relief of 4.3 months (range $0.5-18$; Se 1.03). The average duration of relief and success frequency remained constant after each subsequent radiofrequency treatment. Of the 50 total patients, there was only 1 reported complication, specifically, transient thigh numbness which resolved after one week.

Conclusions: Repeated pulsed and continuous radiofrequency ablation of the lumbar dorsal root ganglion/segmental nerve shows promise to be a safe and effective long-term palliative management for lumbosacral radicular pain in some patients.

Key words: Pulsed radiofrequency lesioning, dorsal root ganglion, segmental nerve, continuous radiofrquency elsioning, chronic lumbosacral radicular pain

Pain Physician 2011; 14:371-376 


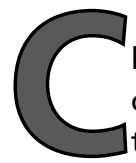

hronic lumbosacral radicular pain is a common source of radiating leg pain seen in the pain management setting. The problem is frequently managed with a combination of phamaceuticals, physiotherapy, and periodic epidural depo-corticosteroid injections. More refractory cases may be successfully managed by spine surgery or spinal cord stimulation. Despite these options for treatment, some patients may not obtain adequate relief, nor are they optimal candidates for any of these therapeutic interventions.

Radiofrequency (RF) has been used to treat chronic radicular pain for over 30 years. Continuous radiofrequency (CRF) lesioning adjacent to the dorsal root ganglion (DRG) for cervical radicular pain has been demonstrated to have efficacy over sham in several trials, but there has been mixed results in the lumbar region (1-3). Several retrospective studies have suggested efficacy for pulsed radiofrequency (PRF) lesioning for lumbosacral radicular pain (4-6). In a prior prospective pilot study, we demonstrated that PRF or PRF in combination with CRF treatments of the lumbar dorsal root/segmental nerves are neurologically safe and well tolerated (7). Patients in the PRF treatment group, as well as the group treated with PRF followed immediately by CRF, reported clinically significant analgesic effects lasting on average 3.18 months and 4.39 months respectively. Even though we were unable to show a statistically significant difference in the time sensitive success between these 2 groups, we felt that PRF in combination with CRF may offer a longer duration of relief that may be clinically beneficial. The difference in duration of action between the 2 modes has been suggested in experimental models. Preclinical studies of PRF and isothermal CRF on impulse propagation and synaptic transmission in hippocampal cell cultures have shown a transient effect on evoked synaptic activity with PRF compared to a lasting effect caused by CRF (8). The premise for the use for continuous radiofrequency lesioning is to produce a partial lesion in the DRG/segmental nerve, so as to preferentially disrupt nociception while avoiding significant sensory deficit.

While there are multiple reports on the potential efficacy of PRF for a variety of painful conditions, the majority does not report on the outcome of repeat application after the analgesic effect is lost (9-13). Indeed, the reproducibility of the therapeutic effects of CRF neurotomy for lumbar facetogenic pain has only recently been assessed $(14,15)$. Radiofrequency of the lumbar DRG for treatment of radicular pain stemming from a multitude of disease processes has been employed in practice for greater than 30 years and is known to have time-limited analgesic success (16). We therefore sought to determine the safety, rate of success, and duration of relief with serial lumbar PRF followed immediately by CRF lesioning of the DRG/segmental nerves (SN) in patients with chronic radicular pain.

\section{Methods}

The Beth Israel Deaconess Medical Center Institutional Review Board approved this study. A neutral party (CWD) with respect to the final outcome used a computerized database to identify all patients of 2 physicians at a single center who underwent PRF/CRF of the lumbar DRG/SN for radicular pain from January 21, 2003 through March 1, 2010. All patients included in the study had detailed history and physical exams, multi-modal radiographic imaging, and diagnostic injections. The inclusion/exclusion criteria for the study used were similar to previous work (7). The inclusion criteria were as follows:

- A greater than 6-month history of segmental pain of lumbosacral origin radiating from the back into the lower extremity

- $\quad$ Age over 18 years

- Absence of a progressive motor deficit

- Absence of a significant sensory deficit

- Unsatisfactory pain control with oral pharmacotherapy and physical therapy

- No urgent need for open surgical intervention

- Magnetic resonance imaging evidence of nerve root involvement

- A response to epidurally administered depo-steroid of one month or less

- Documented complete relief of radicular symptoms following low-volume segmental nerve block

- A $50 \%$ reduction in pain intensity on a numeric rating pain scale (NRS) after one PRF/CRF treatment of the lumbosacral DRG/SN followed by at least a subsequent treatment after the analgesic effects had dissipated.

- The exclusion criteria for the study included the following:

- Evidence of significant neurological deficit, which included a progressive motor deficit and dense sensory loss

- A less than $50 \%$ reduction in NRS after one PRF/CRF treatment of the lumbosacral DRG/SN

- Hypersensitivity to injected materials: local anes- 
thetic, contrast, depo-corticosteroids

- Coagulopathy

- Significant psychopathology

- Pending workers' compensation claims

- Pregnancy.

The response to each repeat radiofrequency treatment was compared to the initial successful treatment and was categorized as successful (by a $50 \%$ reduction of NRS or better) or failure (less than $50 \%$ reduction in NRS). Each patient record was used to determine the duration of relief for each serial treatment as well as for any adverse neurological events (numbness, weakness, or increase in pain level).

The techniques for performing diagnostic segmental nerve root blocks and PRF/CRF of the lumbosacral DRG/SN have been previously described by Simopoulos et al (7). PRF was done at $42^{\circ} \mathrm{C}$ for 120 seconds. During each second of a PRF treatment, 2 bursts of 20-milliscecond intervals delivered alternating current $(500,000$ $\mathrm{Hz}$ ) to the surrounding tissue. The active 20-millisecond phase was followed by a 480-millisecond phase for heat dissipation. The voltage output was 45 . Upon completion of the PRF, CRF was then applied to the maximum tolerated temperature that created a heat sensation in the lower extremity that was concordant with the patient's radiating pain pattern. This temperature averaged $56^{\circ} \mathrm{C} \pm 8^{\circ} \mathrm{C}$ for 60 seconds. No local anesthetic agent was injected prior to the application of CRF or PRF. The radiofrequency lesion generator (RFG-3C Plus; Radi- onics, Inc., Burlington, MA) was used for all lesions. A C-arm (Siremobil, 2000, Siemens AG, Munich, Germany) fluoroscopy machine was used for visualization during the sterile placement of the RF electrode $(22-G, 10 \mathrm{~cm}$ needle, with a curved $10 \mathrm{~mm}$ active tip, Radionics, Burlington, MA or Neurotherm, UK). Once the electrode was appropriately positioned (Figs. 1 \& 2), the needle stylet was then replaced by the radiofrequency probe (SMK-TC 5, Radionics, Burlington, MA). The final positional requirements treated were as follows: 1 ) sensory stimulation $(50 \mathrm{~Hz}$ ) threshold under $0.6 \mathrm{~V}$ (range was 0.3-0.6 V) that created paresthesia concordant to the usual chronic pain distribution; the stimulation must be felt down to the ankle/foot for $L 4 / L 5 / S 1$, groin for $L 1 / 2$, and anterior thigh to knee for L3.2) Motor stimulation (2 HZ) was greater than 1.5 times the sensory stimulation threshold. (3) Impedances were checked to ensure a complete electrical circuit and ranged from 200-400 $\Omega$. (this is a Greek capital omega)

\section{Results}

There were 50 patients identified through record review that underwent pulsed and continuous radiofrequency lesioning of the lumbar dorsal root ganglion/segmental nerves through the specified period who met the inclusion/exclusion criteria. Of these, 26 women and 24 men were included with a mean age of 62 (range 25-86). Twenty-six patients received one level of therapy, 15 patients received 2 levels, and 9 patients received 3 levels. Forty-two treatments were unilateral
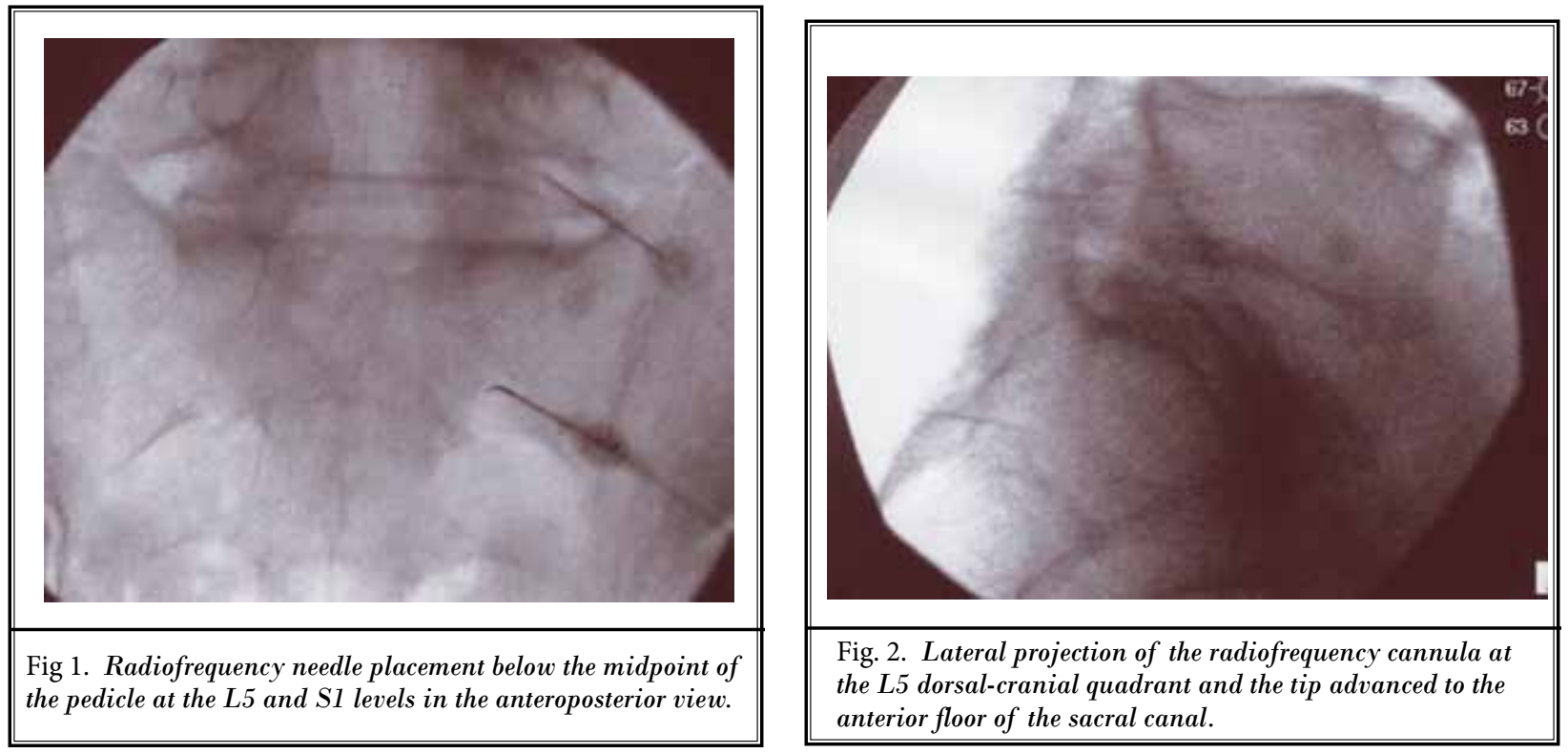
Table 1. Success and duration of relief of pulsed and continuous radiofrequency serial treatments of the lumbar dorsal root ganglion/segmental nerves for radicular pain

\begin{tabular}{|l|c|c|c|c|c||}
\hline \multicolumn{1}{|c|}{ RFN Total \# Treatments } & $\mathbf{1}$ & $\mathbf{2}$ & $\mathbf{3}$ & $\mathbf{4}$ & $\mathbf{5 +}$ \\
\hline Outcome & & & & & \\
Total patients treated & 50 & 40 & 28 & 20 & 18 \\
$\quad$ Successes (\%) & $50(100 \%)$ & $38(95 \%)$ & $27(96 \%)$ & $19(95 \%)$ & 1 \\
Failures & 0 & 2 & 1 & $4.4)$ \\
& & & & 1 \\
\hline Duration of relief (mo) & $4.5(0-24)$ & $4.7(0-24)$ & $4.5(0-19)$ & $4.4(0.5-18)$ & $4.3(0.5-18)$ \\
\hline
\end{tabular}

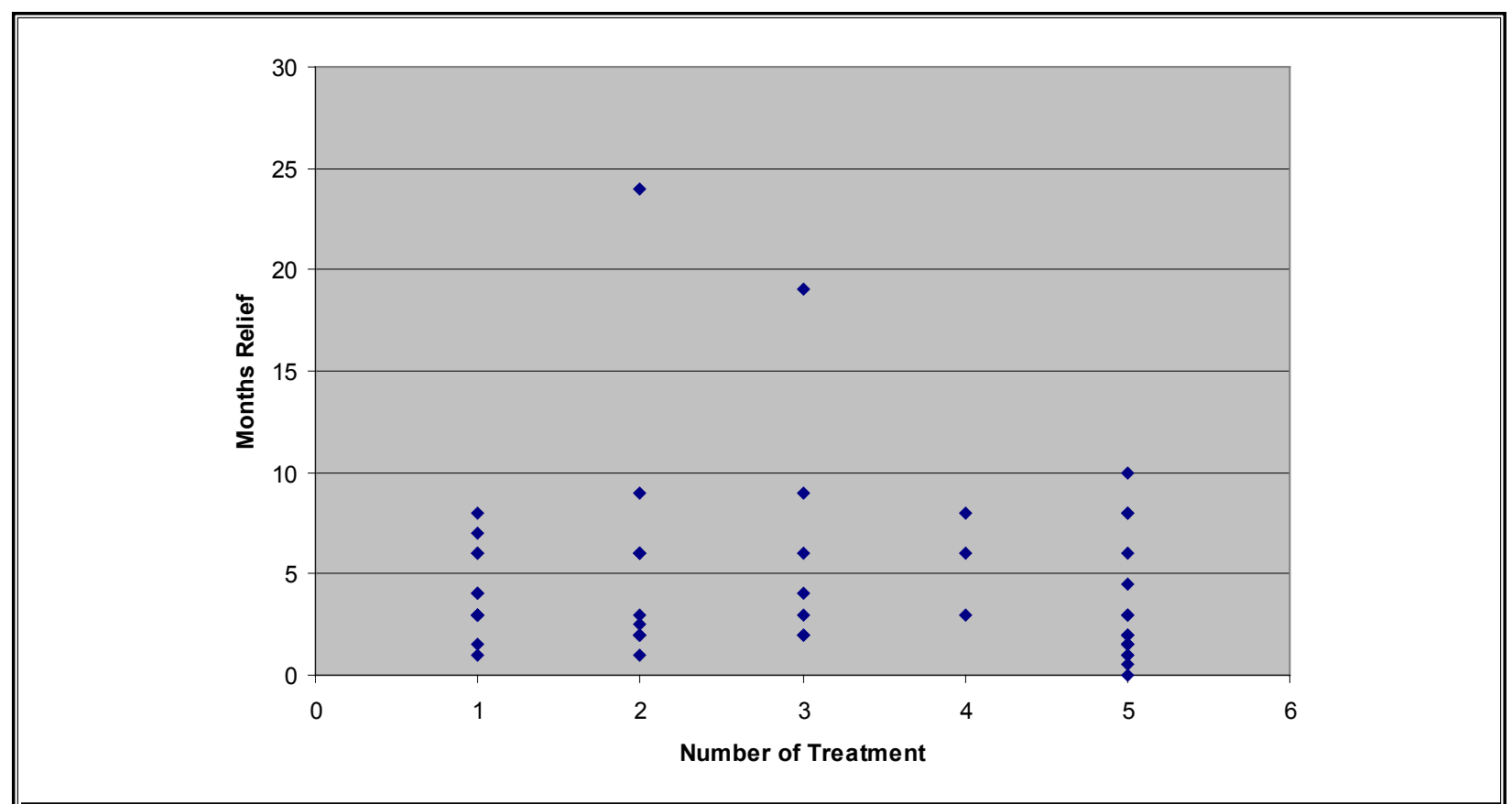

Fig 3. Number of treatments and months relief of lumbar RF-DRG. Each point can represent more than one patient because of overlapping data.

while 8 patients received bilateral therapy. Of the 50 patients, 48 received treatment at the $L 3$ level or lower. Only 2 patients were treated at the L1 and L2 level.

Table 1 summarizes the success/failure of serial PRF/CRF treatments of the lumbar DRG/SN. The mean duration of relief for the 40 patients receiving 2 treatments was 4.7 months (range $0-24, \mathrm{~S}_{\mathrm{e}}$ [standard error] 0.74). Twenty-eight patients had 3 treatments with an average duration of relief of 4.5 months (range 0-19 months; $S_{e} 0.74$ ). Twenty patients receiving 4 treatments had an average duration of relief of 4.4 months (range $0.5-18 ; \mathrm{S}_{\mathrm{e}} 0.95$ ) and 18 patients with 5 or more treatments had an average duration of relief of 4.3 months (range $0.5-18 ; S_{e} 1.03$ ). The duration of relief and success frequency remained constant after each subsequent RF treatment with no apparent trend toward an additive or dwindling benefit. Of the 50 total patients, there was only one reported complication: transient thigh numbness following a second treatment, which resolved after one week. There were no objective neurologic findings documented on follow-up visit. There were no reported long-term neurological deficits, including motor loss, hyposensitivity, or dysesthesias in any of the patients reviewed.

Figure 3 graphically depicts the scatter of relief for patients in the serial treatment groups. Aside from 2 
patients who derived marked prolonged benefit after the second and third treatment, there is consistent response of relief of 10 months as the upper limit. The 2 patients with prolonged benefit presented for repeat treatment and both fell into the range of 10 months relief or less.

\section{Discussion}

The present study supports the neurological safety of serial radiofrequency treatments consisting of both PRF and CRF of the DRG/SN over 2 years in a group of 18 patients (Table 1). However, preclinical data would have suggested otherwise. It is reasonably accepted that the irreversible tissue destruction threshold for nerve fibers by CRF is thought to range between $45-50^{\circ} \mathrm{C}$. CRF application to spinal nerve roots in dogs causes myelin breakdown, nerve cell necrosis, axon loss, and hemorrhage at temperatures of $45^{\circ} \mathrm{C}$ or greater (17). On the other hand, PRF has been speculated to have a nondestructive, or neuromodulatory role, based on the isothermal nature of this therapy. The nondestructive nature of PRF has recently been challenged in that very high electric fields are capable of disrupting neuronal cell architecture and function (18). Recent ultrastructural changes in axons following exposure to PRF include mitochondrial swelling and disruption of the cytoskeleton by disorganization of microtubules and microfilaments (19). Therefore, using both modalities on neuronal tissue, one would expect in the short, and potentially in the long run, the presence of dyesthesias and hypoesthesias that were not observed in the present study. Painful dysesthesias were likely avoided by applying CRF to a maximum tolerated level as reported previously (7). Furthermore, enough neurons/axons were left intact to allow for normal sensation so as to avoid dermatomal numbness. There is probably modest insult to the neural tissue with PRF/CRF combination that resolves in the interval before the subsequent treatment, so as to avoid additive insult to neurons/ axons. Light microscopy data demonstrates that endoneurial edema and fibroblast activation, as indicated by collagen deposition, resolves after day 21 following exposure to PRF (20).

The benefits of both CRF and PRF for the treatment of chronic radicular pain have been described but both are not a cure and have time-sensitive success $(16,7)$. It is well known that the benefits of CRF or PRF dwindle and the treatment will need to be repeated for chronically painful conditions in most cases. The authors are not aware of any reports specifically addressing the outcomes of radiofrequency used as a maintenance therapy for radicular pain. In the present study, the apparent success rate for repeat PRF/CRF treatments is successful in at least $95 \%$ of patients. The average duration of relief remains constant at just over 4 months. This is in agreement with prior prospective reports of analgesic benefit (7). The analgesic effect of radiofrequency for radicular pain is significantly less than for facet pain (10 months) emphasizing the likely prolonged benefit of thermal neurolysis (14). Of note, 2 patients obtained prolonged benefit from CRF/PRF therapy, but have had recurrent symptoms, and when treated had 10 months benefit or less. These dramatic exceptions are difficult to explain but have been reported by others as well (11).

One of the inclusion criteria used in this study was at least a $50 \%$ reduction of pain intensity. Such a criterion has been shown to correlate with a high degree of patient satisfaction and thereby increase the chance that a serial treatment will be requested $(14,20)$. Even with a clinically significant reduction in pain intensity, 40 out of 50 initial patients sought a second treatment following the first treatment, followed by 28 out of 40 for the third treatment. The most common reason for this is the duration of analgesia was too brief for patients to accept radiofrequency as a maintenance therapy. In addition, other treatments for radicular pain such as decompression or spinal cord stimulation were also sought by patients, but the authors did not seek out these quantitative details since this was not within the goals of the present study. This is in contrast to facetogenic pain whereby radiofrequency is the primary treatment for long-term relief and patients are more likely to return for repeat treatments.

A small but consistent number of patients failed to obtain relief on subsequent treatments. The reasons for the lack of effect may include placebo response to the first RF treatment, subsequent technical failure, a different structural cause of pain with radiating symptoms, and a lack of response to RF because of progressive dysfunction of the affected DRG/SN. The technical aspects of the procedure are in most cases easily repeatable. In the majority of cases, if another anatomic structure is causing pain, the axial component becomes more prevalent and a different treatment ensues. We therefore feel that the clinical dissatisfaction for patients following PRF/CRF treatment of the DRG/SN is weighted more on the duration of relief rather than the reduction in pain intensity.

There are limitations as well as strengths to the 
present study. The study is retrospective without a control group, and therefore no direct statements can be made about analgesic efficacy. Furthermore, there is a small sample size with no secondary outcome measures on function. On the other hand, the clinical outcome of repeat $\mathrm{RF}$ is driven by patient satisfaction and we believe it is directly clinically relevant. The long-term follow-up and multiple repeat treatments indirectly suggest analgesic efficacy. Other treatments such as more invasive lumbar spine surgery have shown exponentially declining success ranging from $80-98 \%$ with the initial surgery, declining to $5 \%$ by the fourth surgery (22). This epidemiologic data suggests that patients with radiating leg pain from the lumbar spine are not so predisposed to placebo or operant conditioning from serial treatments. All patients entered into the study had specific inclusion/ exclusion as well as treatment criteria. The procedures were carried out by experienced physicians and the data were collected by a party neutral to the final outcome.

\section{Conclusion}

Our findings in the present study suggest that PRF/ CRF of the DRG/SN appears to be a useful and safe treatment in a serial fashion for chronic radicular pain. A select group of patients may be managed successfully long-term with multiple treatments. The role of this modality may be for the patient who is not a candidate or does not desire more invasive surgical options. Finally, there are no reports of RF treatments causing devastating neurological complications, unlike transforaminal depo-corticosteroid injections (21).

\section{References}

1. Van Kleef M, Liem L, Lousberg R, Barendse G, Kessels F, Sluijter M. Radiofrequency lesion adjacent to the dorsal root ganglion for cervical brachial pain: A prospective double blind randomized study. Neurosurgery 1996; 38:1127-1132.

2. Van Wijk RMAW, Geurts JWM, Wynne $\mathrm{HJ}$. Long-lasting analgesic effect of radiofrequency treatment of the lumbar dorsal root ganglion. J Neurosurg 2001; 94:227-231.

3. Geurts JWM, van Wijk RMAW, Wynne HJ, Hammink E, Buskens E, Lousberg R, Knape JTA, Groen GJ. Radiofrequency lesioning of dorsal root ganglia for chronic lumbosacral radicular pain: A randomized double-blind, controlled trial. Lancet 2003; 361:21-26.

4. Teixeira A, Grandinson M, Sluijter ME. Pulsed radiofrequency for radicular pain due to a herniated intervertebral disc-an initial report. Pain Pract 2005; 5:111-115.

5. Abejon D, Garcia-del-Valle S, Fuentes ML, Gomez-Arnau Jl, Reig E, van Zundert J. Pulsed radiofrequency in lumbar radicular: Clinical effects in various etiological groups. Pain Pract 2007; 7:21-26.

6. Martin DC, Willis ML, Mullinax LA, Clarke NL, Hamburger JA, Berger $\mathrm{IH}$. Pulsed radiofrequency application in the treatment of chronic pain. Pain Pract 2007; 7:31-35.

7. Simopoulos TT, Kraemer J, Nagda JV, Aner M, Bajwa ZH. Response to pulsed and continuous radiofrequency of the dorsal root ganglion and segmental nerves in patients with chronic lumbar radicular pain. Pain Physician 2008; 11:137-144.

8. Cahana A, Vutskits L, Muller D. Acute differential modulation of synaptic transmission and cell survival during exposure to pulsed and continuous radiofrequency energy. J Pain 2003; 4:197-202.

9. Brennan L, Fitzgerald J, McCrory C. The use of pulsed radiofrequency treatment for chronic benign pancreatitis pain. Pain Pract 2009; 9:135-140.

10. Kim YH, Lee CJ, Lee SC, Huh J, Nahm FS, Kim HZ, Lee MK. Effect of pulsed radiofrequency for postherpetic neuralgia. Acta Anaesthesiol Scand 2008; 52:11401143 .

11. Rhame EE, Levey KA, Gharibo CG. Successful treatment of refractory pudendal neuralgia with pulsed radiofrequency. Pain Physician 2009; 12:633-638.

12. Philip CN, Candido K, Joseph NJ, Crystal GJ. Successful treatment of meralgia paresthetica with pulsed radiofrequency of the lateral femoral cutaneous nerve. Pain Physician 2009; 12:881-885.

13. Ramanavarapu V, Simopoulos TT. Pulsed radiofrequency of the lumbar dorsal root ganglia for chronic post-amputation stump pain. Pain Physician 2008; 4:561566.

14. Schofferman J, Kine G. Effectiveness of repeated radiofrequency neurotomy for lumbar facet pain. Spine (Phila Pa 1976) 2004; 29:2471-2473.

15. Rambaransingh B, Stanford G, Burnham R. The effect of repeated zygapophysial joint radiofrequency neurotomy on pain, disability, and improvement duration. Pain Med 2010; 11:13431347 .

16. Malik M, Benzon $H$. Radiofrequency applications to dorsal root ganglion. Anesthesiology 2008; 109:527-542.

17. Smith HP, McWhorter JM, Challa VR. Radiofrequency neurolysis in a clinical model. J Neurosurg 1981; 55:246-253.

18. Cosman ER Jr, Cosman ER Sr. Electric and thermal field effects in tissue around radiofrequency electrodes. Pain Med 2005; 6:405-424.

19. Erdine S, Cosman ER Sr, Cosman ER Jr. Ultrastructural changes in axons following exposure to pulsed radiofrequency fields. Pain Pract 2009; 9:407-417.

20. Farrar JT, Young JP, LaMoreaux I, Werth J, Poole RM. Clinical importance of changes in chronic pain intensity as measured on an 11-point numerical rating scale. Pain 2001; 94:149-58.

21. Houten Jk, Errico TJ. Paraplegia after lumbosacral nerve root block. Spine ] 2002; 2:70-75.

22. Nachemson AL. Evaluation of results in lumbar spine surgery. Acta Orthop Scand Suppl 1993; 251:130-133. 